\title{
A new sensor-based self-configurable bandstop filter for reducing the energy leakage in industrial microwave ovens
}

\author{
F J Clemente-Fernández, J Monzó-Cabrera, J L Pedreño-Molina, A J Lozano- \\ Guerrero, J Fayos-Fernández and A Díaz-Morcillo \\ Departamento de Tecnologías de la Información y Comunicaciones, Universidad \\ Politécnica de Cartagena. Plaza del Hospital, 1, Edificio Antigones. 30202, Cartagena, \\ Murcia, Spain \\ E-mail: francisco.clemente@upct.es
}

\begin{abstract}
In this work a new sensor-based self-configurable waveguide bandstop filter that uses a combination of metallic irises and reconfigurable posts for reducing the energy leakage in industrial microwave ovens is presented and validated through a procedure fully based on measurements. Several optimization and reconfiguration alternatives of the moving posts such as genetic algorithms and parametric sweeps are assessed. Results show that good attenuation values can be obtained for all the analyzed scenarios. In particular, genetic algorithms are shown as the best search strategy. Design and optimization times are also reduced when using the proposed filter compared to computer simulations.
\end{abstract}

Keywords: bandstop filter; microwave heating; microwave sensor; self-configurable

PACS: 84.40.Az Waveguides, transmission lines, striplines. 84.30.Vn Filters.

Submitted to: Measurement Science and Technology

\section{Introduction}

Industrial microwave-heating ovens are extensively used in many industrial applications [1]. In particular, the processing of materials by means of continuous-flow industrial microwave ovens placed at some point in the production line is a fact with growing presence in industry. This kind of microwave ovens often employs open-ended waveguide ports in order to allow the continuous flow of the processed material through the multimode heating cavity by means of a carrying belt, as depicted in figure 1. The high power levels irradiated within such devices (usually several kilowatts) may be harmful for the operators who are in direct contact with the microwave equipment due to microwave energy leakage through the open ports [2].

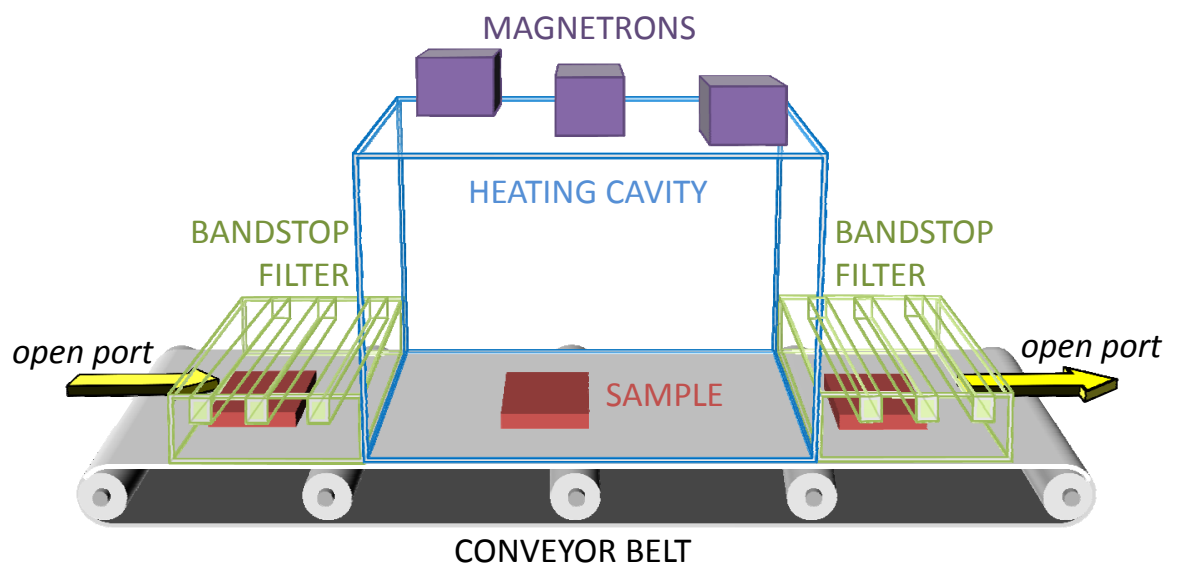

Figure 1. Simplified model of a continuous flow industrial microwave oven. 
Waveguide bandstop filters are usually employed in order to prevent these possible hazardous effects on human tissues. The scientific literature on this topic is very extensive: this sort of filters may be based on short-circuited stubs [3], resonant structures [4, 5], metallic posts [6-13] or irises [14-19] to name a few. In particular, if we focus on filters that make use of any kind of irises or posts, some previous work can be found in technical literature. The basis of the influence of cylindrical posts in waveguides was established more than forty years ago [6], leading to some contributions over the last twenty years. Thus, in [7] the effect of multiple inductive posts in a rectangular waveguide was studied. The first filter application that made use of cylindrical posts was presented in [8], and from that moment several alternatives for modeling and designing these filters were developed (equivalent circuits [9], full wave modeling [10] or CAD design [11]), ending in contributions such as [12] and [13], where posts are employed to implement bandstop and bandpass filters, respectively.

Considering the use of irises to implement filters, few contributions can be found, particularly if we focus on bandstop filters. The first contribution, mainly theoretical [14], was followed by some studies dealing with cavities with asymmetric irises [15], CAD design [16, 17], multiaperture irises [18], or slotted strips [19].

Regarding industrial microwave heating, some alternatives to deal with this leakage problem have been proposed, usually based on corrugated and doubly corrugated reactive filters [20] which reflect back the energy escaping from the applicator. The study of this kind of structures has been carried out in $[21,22]$ where both singly and doubly corrugated filters are analyzed, designed and experimentally validated. However, this study is performed in the non-realistic scenario where no material is placed inside the filter. Results when designing bandstop filters in this case may be different from those obtained when a sample of a material is inside the structure. In fact, it is shown in [23] that these filters do not work properly with certain materials depending on the dielectric properties of the sample.

In this work a new patented waveguide bandstop filter based on a combination of metallic irises and automatically reconfigurable posts is evaluated [24, 25]. Some high power microwave devices such as auto-tuners also make use of reconfigurable posts in order to match impedances between the magnetron and the load [26]. In our case, the reconfigurable posts allow us to optimize the bandstop response of the filter, i.e. to maximize the attenuation, just by applying a self-configuration and optimization procedure. In addition, a sensing system based on a coaxial monopole antenna is also used in order to measure the leaked energy and configure the filter so that this leaked energy is reduced. In this way, several optimization schemes based on genetic algorithms (GA) and parametric sweeps have been applied to evaluate the behavior of the filter along with the sensing system for different scenarios.

\section{System description}

\subsection{New bandstop filter}

One of the main disadvantages of traditional corrugated filters is that they have a fixed structure, which makes them appropriate for just one type of process, defined by the type and size of the processed material, the microwave feeding system, the microwave oven cavity and the power level. This fact implies that any change in the productive process forces a redefinition and remanufacturing of the employed filters.

On the contrary, the presented bandstop filter is able to reconfigure itself when some of the parameters of the process (e.g. the material or the feeding system of the cavity) are changed, with the automatically tunable metallic posts and measurement system, in order to reduce the leaked energy under the new conditions.

In particular, the manufactured prototype, which can be seen in figure 2 , is a rectangular waveguide with cross section of $50 \mathrm{~cm} \times 6 \mathrm{~cm}$ and length of $50 \mathrm{~cm}$, which are plausible values for this kind of devices that deal with thin materials at the frequency of $2.45 \mathrm{GHz}$. This manufactured filter employs ten rows of metallic screws with diameter of $19.5 \mathrm{~mm}$ and ten screws per row. Attached to these metallic screws, ten stepper motors are used to screw on and off the metallic posts in a single row to the structure of the filter automatically. Figure 3 shows the actual manufactured prototype along with a detailed view of the tunable screws. In addition, eleven metallic irises with width of $2.4 \mathrm{~cm}$ and thickness of $2 \mathrm{~mm}$ are placed in between the rows of posts. It must be remarked that with this configuration, only a row as a whole could be moved at each optimization step. 
Thus, the parameters we are optimizing are the penetration of each post in the waveguide, which control the bandstop response of the filter for each scenario. Each post is optimized independently when optimizing a row configuration during genetic algorithms procedures. However, in the case of using a parametric sweep optimization strategy the penetration of all posts is the same within each row. Further details on this self-configuration and optimization process can be found in section 3 .

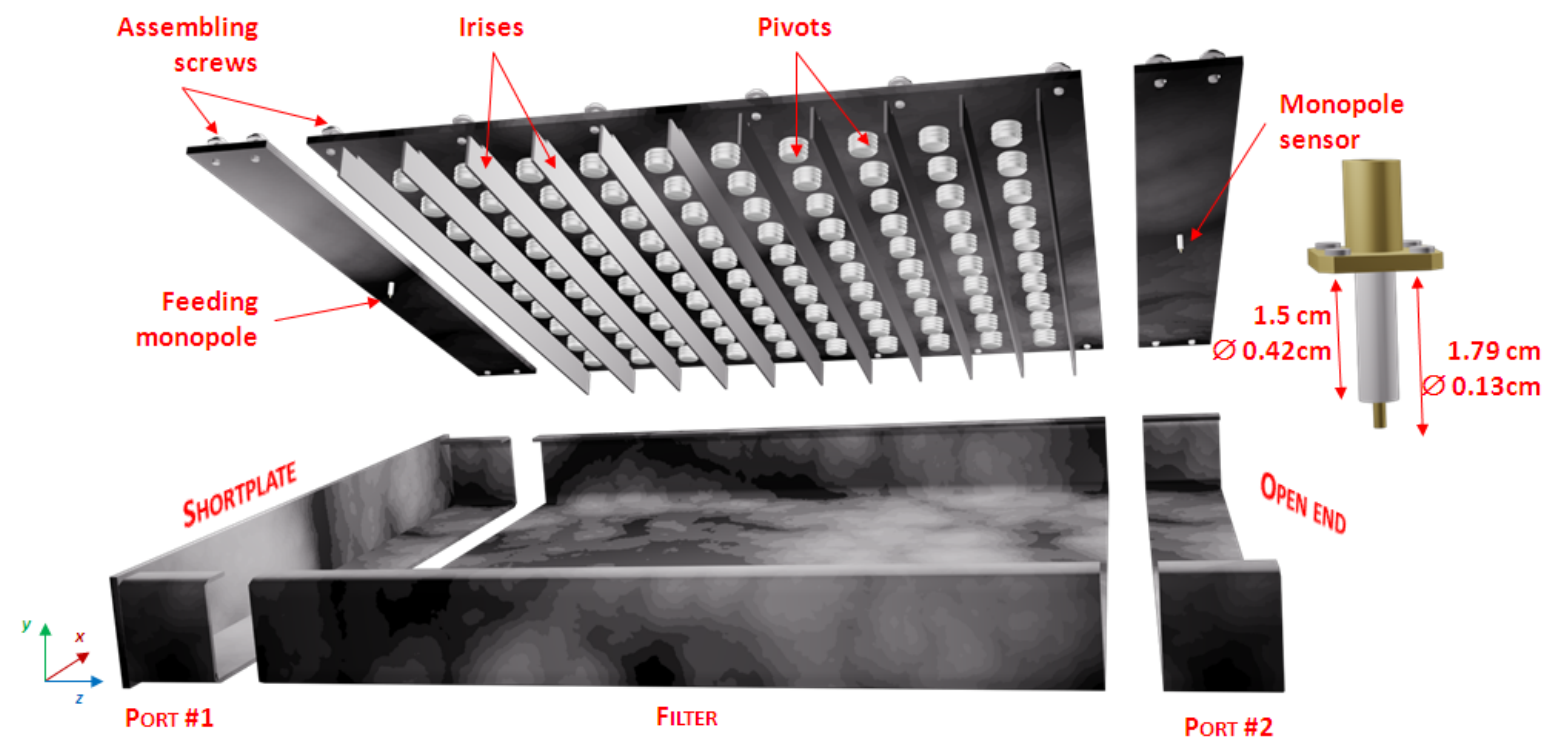

(a)

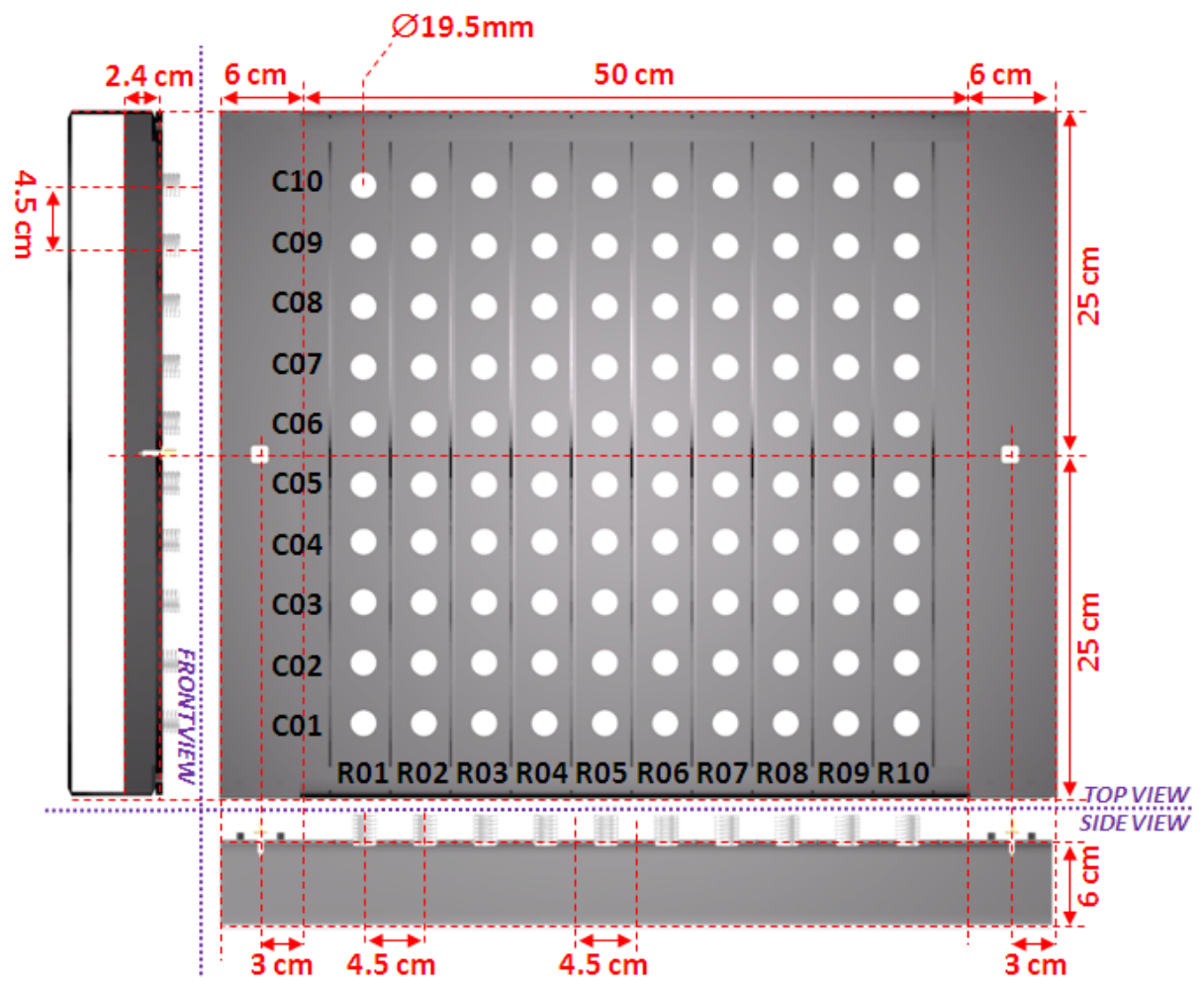

(b)

Figure 2. (a) Exploded view with the monopole antenna used for excitation and sensing purposes and (b) dimensions of the new bandstop filter prototype. 


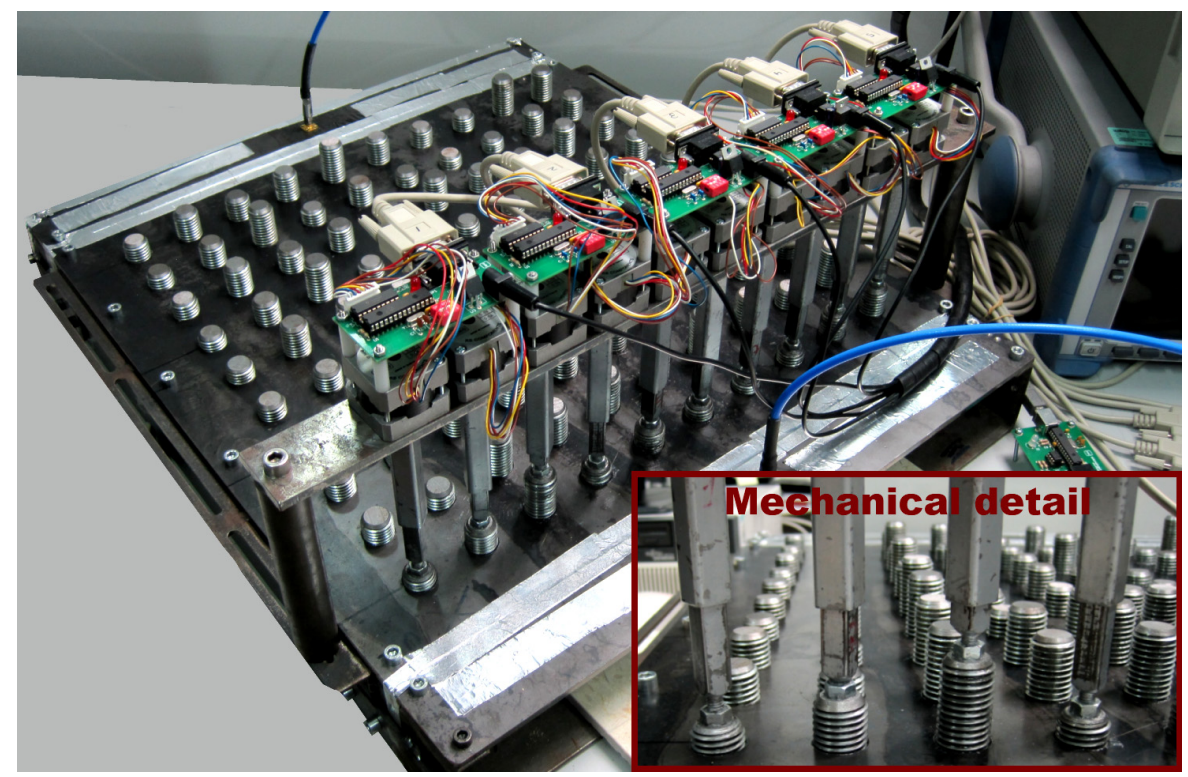

Figure 3. Actual manufactured prototype and mechanical detail of the tunable screws.

\subsection{Sensing and excitation systems}

In order to obtain the performance of the filter for each scenario, it is necessary to measure the power level at the output port of the filter so that each configuration of the posts can be evaluated. Furthermore, in order to perform tests in a laboratory without using an actual industrial oven, it is necessary to feed the microwave power into the filter.

In this way, a coaxial monopole antenna is employed for both purposes. Firstly, for the excitation of the filter a back-short waveguide with length of $6 \mathrm{~cm}$ and the same cross section as the filter is attached to the input port of the filter. The coaxial antenna is centered at the $\mathrm{x}$-dimension of the filter on the top wall of the waveguide and $3 \mathrm{~cm}$ away from the shortplate, as depicted in figure $2 \mathrm{~b}$, which corresponds to the position in which the generated progressive and regressive waves are in phase for the $\mathrm{TE}_{10}$ mode. Furthermore, placing the monopole in this position ensures that the $\mathrm{TE}_{10}, \mathrm{TE}_{30}, \mathrm{TE}_{50}$ and $\mathrm{TE}_{70}$ modes are excited since the antenna is placed at the maximum of the electric field distribution of these propagation modes. Figure 4 shows the simulation scheme of the excitation system consisting of a multimode coaxial to waveguide transition. In this case, port one is placed at the coaxial probe connector whereas port two is placed at the waveguide cross-section that connects to the filter. According to the well-known cut-off frequency of a rectangular waveguide, only 8 modes could propagate at $2.45 \mathrm{GHz}: \mathrm{TE}_{10}, \mathrm{TE}_{20}, \mathrm{TE}_{30}, \mathrm{TE}_{40}, \mathrm{TE}_{50}, \mathrm{TE}_{60}, \mathrm{TE}_{70}$ and $\mathrm{TE}_{80}$. Figure 5 shows the transmission coefficient obtained for each mode when simulating the back-short waveguide excited by the coaxial monopole by means of CST Microwave Studio ${ }^{\circledR}$ software [27]. This transmission coefficient represents the power loss from the coaxial connector to the waveguide port of the transition for each mode. It is shown that all the excited modes, namely $\mathrm{TE}_{10}, \mathrm{TE}_{30}, \mathrm{TE}_{50}$ and $\mathrm{TE}_{70}$, show similar amplitudes at the frequency of $2.45 \mathrm{GHz}$ since the transmission coefficient is very similar for all of them. As expected, the TE modes with even indices $\mathrm{TE}_{20}, \mathrm{TE}_{40}, \mathrm{TE}_{60}$ and $\mathrm{TE}_{80}$ are not excited which is proven by the low transmission coefficient values provided by simulations.

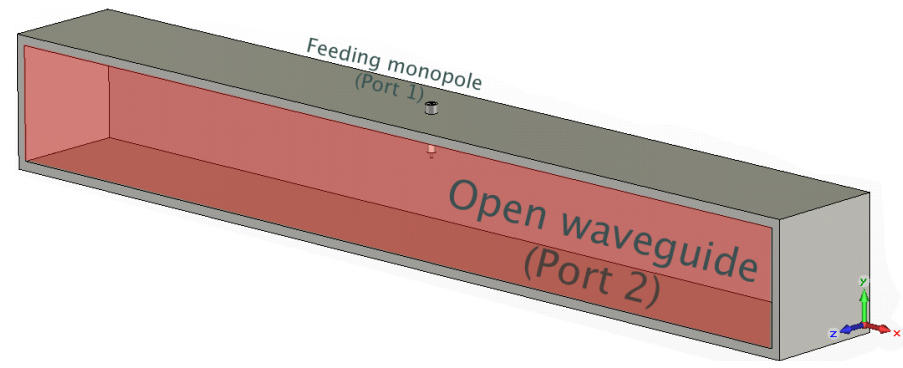

Figure 4. Simulation scheme of the coaxial to waveguide transition functioning as excitation system. 


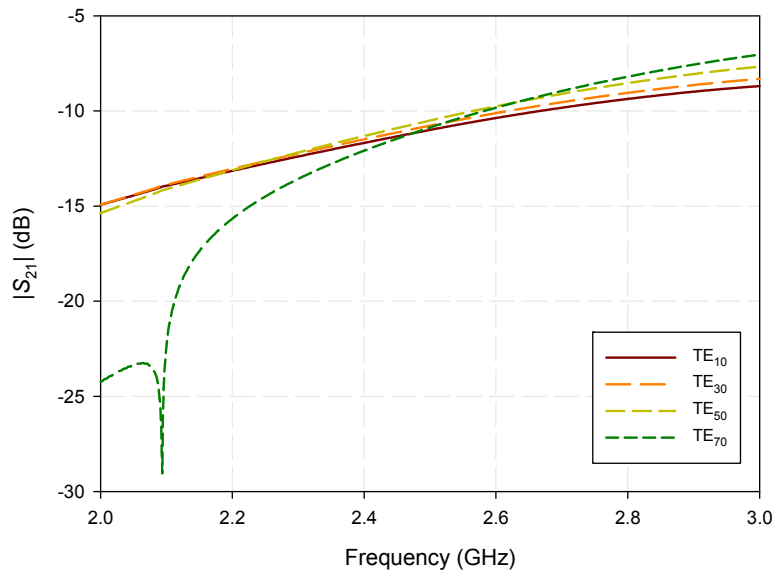

(a)

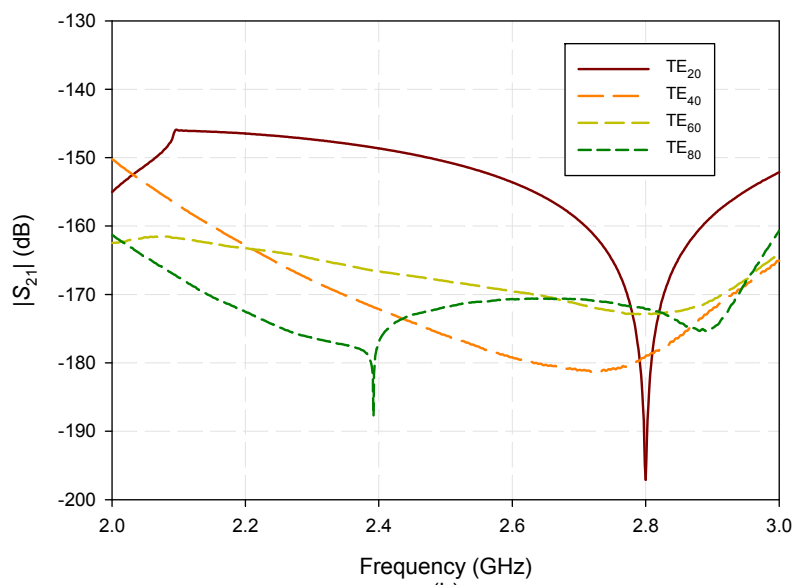

(b)

Figure 5. Transmission coefficient in $\mathrm{dB}$ for all the modes above cut-off in the excitation transition.

(a) Excited modes $\left(\mathrm{TE}_{10}, \mathrm{TE}_{30}, \mathrm{TE}_{50}, \mathrm{TE}_{70}\right)$. (b) Non-excited modes $\left(\mathrm{TE}_{20}, \mathrm{TE}_{40}, \mathrm{TE}_{60}, \mathrm{TE}_{80}\right)$.

For this laboratory tests an Agilent $8648 \mathrm{C}$ signal generator has been employed in order to feed the filter with a $2.45 \mathrm{GHz}$ sine wave with power of $14.5 \mathrm{dBm}$. Since the filter under study is a passive device, its behavior will be quite similar when high power levels at industrial scale are employed. Nevertheless, it is true that several phenomena such as arcing might occur [28] but this is something common to this kind of high power devices and not a particular issue of the proposed filter.

Similarly, another $6 \mathrm{~cm}$ waveguide is placed at the output port of the filter. In this case the waveguide remains open, as in actual working conditions. The coaxial monopole, which in this case works as a sensor measuring the leaked power, is again centered on the top wall of the waveguide at a position which would correspond to the maximum of the electric field distribution of the $\mathrm{TE}_{10}, \mathrm{TE}_{30}$, $\mathrm{TE}_{50}$ and $\mathrm{TE}_{70}$ modes, as seen in figure $2 \mathrm{~b}$. In this way, the filter will be able to deal with modes that have a maximum at that position $\left(\mathrm{TE}_{(2 k-1) 0}\right.$ modes, $\left.k>0\right)$, but not with the rest of the modes. The value of the power at the output port of the filter obtained by the monopole is measured by means of a Rohde \& Schwarz FSP7 spectrum analyzer.

\subsection{Experimental setup}

A general view of the whole system is depicted in figure 6a. As it has been explained earlier in the previous section, the signal generator introduces the microwave energy in the filter through the coaxial monopole placed at its input port. Then, the microwave power travels through the filter to its output port, where it is measured by means of the coaxial monopole connected to the spectrum analyzer, which is plugged into a PC via GPIB. Thus, the computer has a measure of the performance of the filter configuration under evaluation, and will reconfigure the posts by using the stepper motors according to a given optimization scheme, which is discussed in the next section. This work flow is summarized in figure $6 \mathrm{~b}$. MATLAB ${ }^{\circledR}[29]$ has been employed to control the whole process.

As it has been explained earlier, the main objective of this sort of bandstop filters is to minimize the microwave power measured at the output port of the filter, or, what is the same thing, to maximize the attenuation $(\alpha)$ of the microwave power travelling through it. In order to calculate this attenuation, two different measurements have been performed. Firstly, the received power at the output port is measured $\left(P_{0}\right)$ when using a hollow waveguide with the same length as the filter instead of using the filter. This value is compared with the received power measured when the filter is employed $\left(P_{\text {filter }}\right)$, so the attenuation can be obtained according to the expression shown in (1).

$$
\alpha(\mathrm{dB})=P_{0}(\mathrm{dBm})-P_{\text {filter }}(\mathrm{dBm})
$$

When optimizing the filter, the power values are obtained at the single frequency of $2.45 \mathrm{GHz}$. Once the optimization procedure has been performed, a frequency sweep from 2.4 to $2.5 \mathrm{GHz}$ has been carried out for the parametric sweep scenario in order to obtain the bandwidth of the filter. 


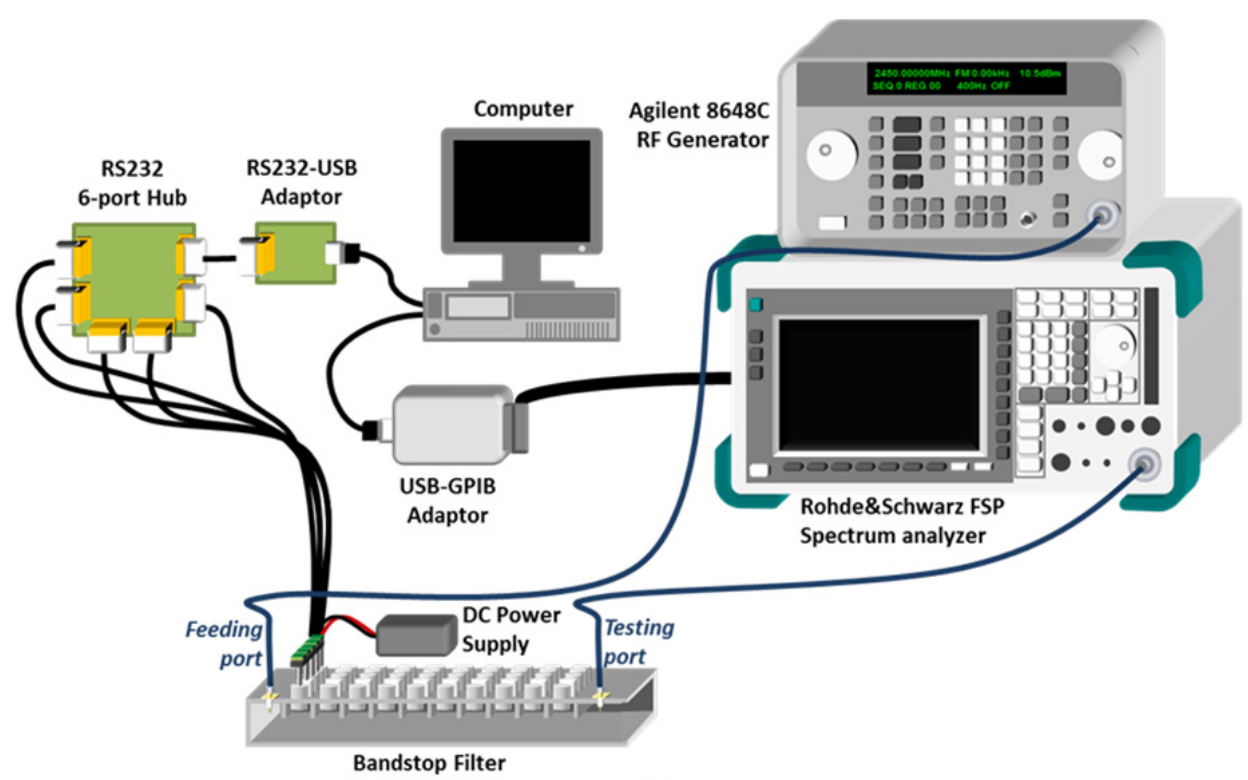

(a)

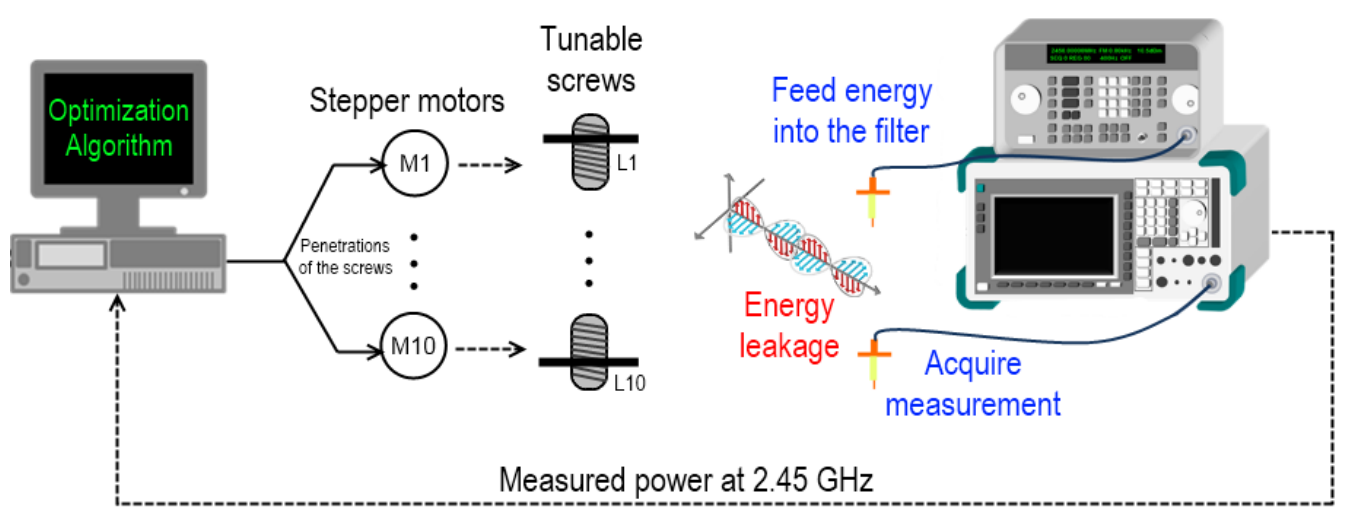

(b)

Figure 6. (a) General diagram and (b) work flow of the experimental setup.

\section{Reconfiguration and optimization procedures}

In order to reduce the microwave leakage when using these filters, two different procedures have been assessed: an optimization scheme based on genetic algorithms and an optimization procedure by using parametric sweeps.

\subsection{Genetic Algorithms}

GA are a powerful optimization tool whose applicability to complex non-linear electromagnetic problems has been proved in previous works [30-32]. In particular, the optimization process when using GA is shown in figure 7.

The process starts by placing the stepper motors at the first row. Then, the first individual generated by the GA is evaluated by measuring the received power at $2.45 \mathrm{GHz}$ by the monopole antenna placed at the output port. It must be pointed out that each individual is represented by the different depths of the ten posts, i.e. a vector of ten values $\left\{p_{1} \ldots p_{10}\right\}$. Then, the performance of this individual is evaluated according to the fitness function $f$, which the algorithm tries to minimize, as follows:

$$
f\left(p_{1} \ldots p_{10}\right)=P_{\text {measured }}(2.45 \mathrm{GHz})[\mathrm{W}]
$$

where $P_{\text {measured }}$ is the power in watts measured by the spectrum analyzer through the coaxial monopole at $2.45 \mathrm{GHz}$. This power is provided by the leakage of $\mathrm{TE}_{10}, \mathrm{TE}_{30}, \mathrm{TE}_{50}$ and $\mathrm{TE}_{70}$ modes at the output port as explained before. Thus, minimizing this power value ensures the reduction of this leakage. 


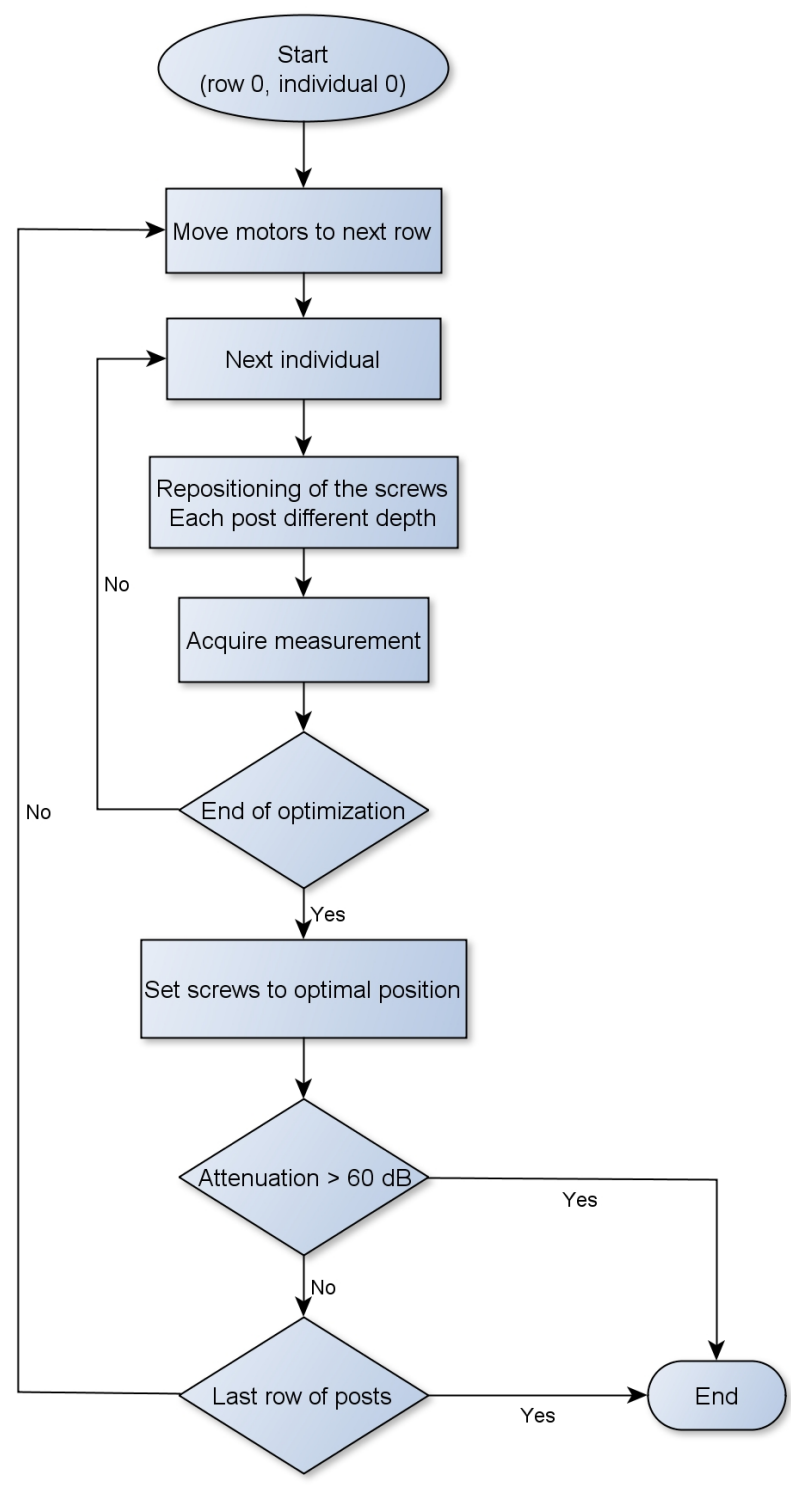

Figure 7. Flowchart of the genetic algorithm optimization.

In this case 10 generations and 10 individuals per generation have been employed. These values for the number of generations and the number of individuals per generation have been chosen after several trials as a trade-off between a good convergence and a reasonable optimization time. Then, the rest of the individuals in a generation are evaluated and those that minimize the fitness function are taken into account to obtain the next generation by applying the crossover and mutation functions [30]. In order to provide genetic diversity from one generation to the next, especially in the first ones, the mutation function adds a random number taken from a Gaussian distribution with mean 0 to each entry of the parent vector. The algorithm shrinks the variance in each coordinate linearly until it reaches 0 at the last generation. In this way it is ensured a proper search for the solution during the first stages of the optimization and a refinement of the solution during the last stages of the process. The process goes on until the last generation is completed and the best individual is chosen. After this, the screws are set to the optimum position manually and the stepper motors are moved to the next row of posts. The optimization procedure is carried out again for this following row. The achieved attenuation is checked after the optimization of each row and the process is stopped if its value is greater than $60 \mathrm{~dB}$, which is a good threshold value taking into account that values over $60 \mathrm{~dB}$ usually ensure a proper reduction of the leakage and guarantee a safe usage of the microwave oven. Figure 8 represents an 
example of optimization of one row showing the best fitness and the mean fitness for each generation. It is shown how the algorithm converges to an optimum value in terms of minimum received power.

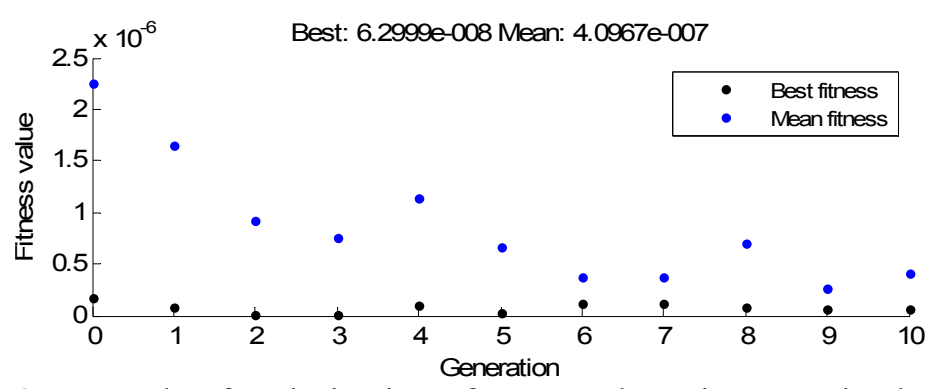

Figure 8. Example of optimization of one row by using genetic algorithms.

Moreover, two different initial alternatives when using GA have been assessed, i.e. starting the optimization process from the row nearest to the input port with all posts totally unscrewed (depth $=0$ $\mathrm{mm}$ ) or starting this optimization process with all posts almost totally screwed (depth $=28 \mathrm{~mm}$ ), and then applying a complete GA optimization stage.

\subsection{Parametric Sweep}

Secondly, once GA optimization has been evaluated, a procedure based on parametric sweeps has been applied. This procedure, whose flowchart is depicted in figure 9, is quite similar to the GA optimization although some important differences need to be considered. Again, the process is started by placing the stepper motors at the first row. In this case, and this is where the main difference lies in, all the posts in a given row are introduced the same depth in the filter. In this way, the depth of the posts is swept from 0 to $28 \mathrm{~mm}$ in steps of $0.5 \mathrm{~mm}$ and the best configuration in terms of minimum received power is chosen. Then, the stepper motors are moved to the next row and the process is repeated. Again, the process is stopped if the attenuation value is greater than $60 \mathrm{~dB}$.

As it can be easily deduced, the number of degrees of freedom when using the parametric sweep approach is less than the case when using GA, since all the post have the same depth. Therefore, in order to refine the results several consecutive parametric sweeps have been applied. The first one is started with all post unscrewed, whereas the following ones are started from the best solution obtained by the previous sweep.

\section{Results and discussion}

\subsection{Genetic algorithms}

Figure 10 shows the attenuation obtained when the optimization process is started with all post being unscrewed, resembling a hollow rectangular waveguide with irises. A general growing trend can be observed when incrementing the number of optimized rows, which seems logical since the optimization of each row starts from a point in which the attenuation is minimal. From this figure, one can conclude that the isolating effect of the irises on the filtering capabilities of the structure is minimum but, when combined with the tuning screws, good attenuation values can be obtained even for big apertures such as the one used in this work.

It can also be seen that the goal of obtaining an attenuation value greater than $60 \mathrm{~dB}$ has been achieved after the optimization of the eighth row, in particular $60.34 \mathrm{~dB}$. In table 1 , the final penetration of the posts after the optimization process is shown. The penetration of the screws placed at rows 9 and 10 is zero since no optimization was longer necessary for them. 


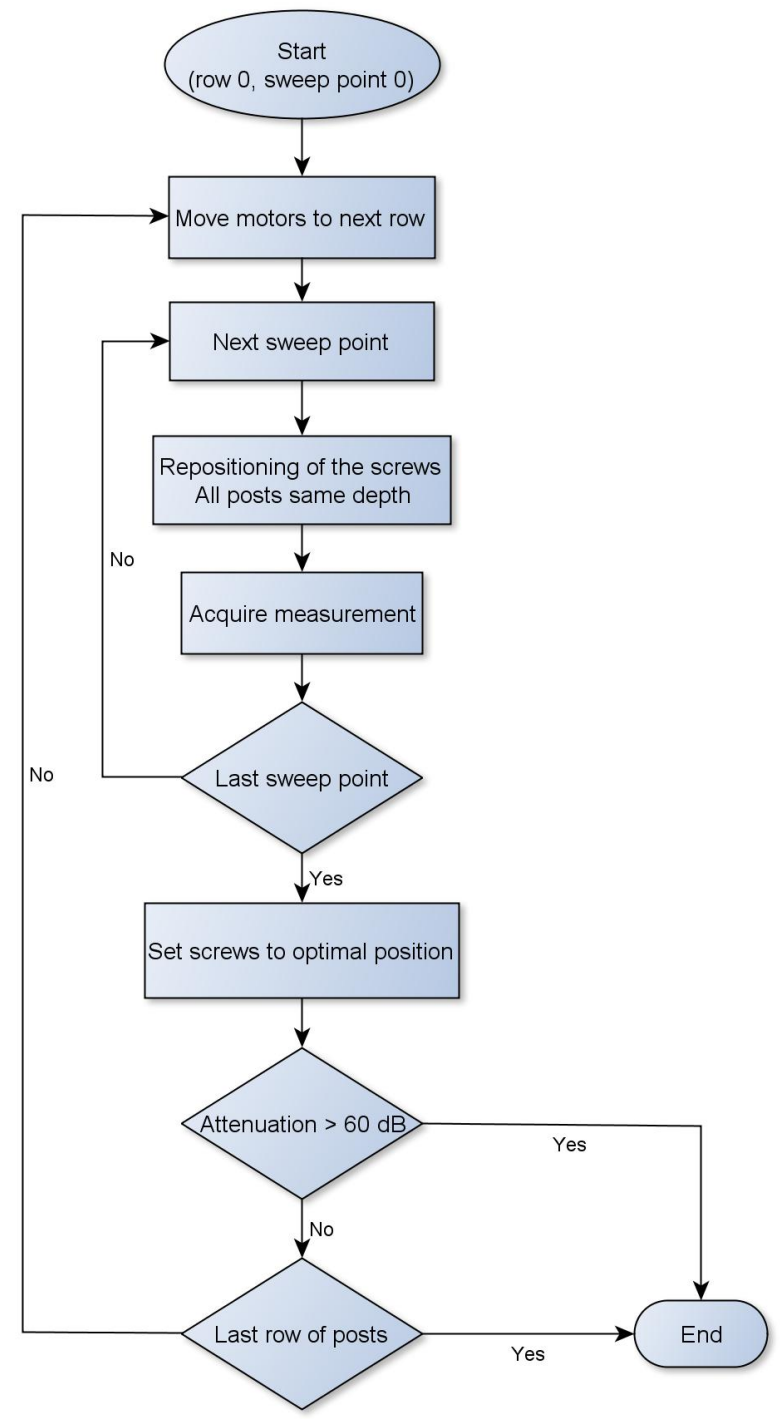

Figure 9. Flowchart of the parametric sweep stage.

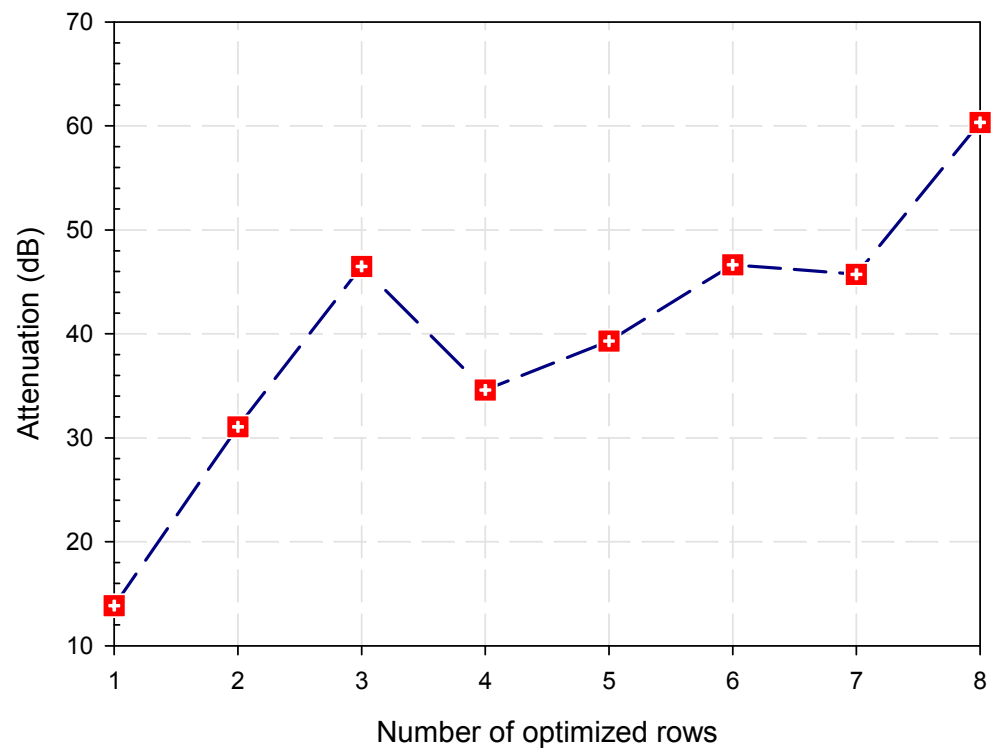

Figure 10. Attenuation in $\mathrm{dB}$ at $2.45 \mathrm{GHz}$ when using GA starting with unscrewed posts. 
Table 1. Penetration of the posts in $\mathrm{mm}$ when using GA starting with unscrewed posts.

\begin{tabular}{c|cccccccccc}
\hline \multicolumn{10}{c}{ Input port } \\
\hline & Col. 1 & Col. 2 & Col. 3 & Col. 4 & Col. 5 & Col. 6 & Col. 7 & Col. 8 & Col. 9 & Col. 10 \\
\hline Row 1 & 26.61 & 27.43 & 27.37 & 18.35 & 26.11 & 20.86 & 2.19 & 25.20 & 13.75 & 16.39 \\
Row 2 & 22.81 & 5.41 & 26.87 & 24.06 & 19.46 & 8.23 & 26.87 & 7.12 & 22.80 & 15.90 \\
Row 3 & 26.82 & 13.59 & 24.28 & 0.89 & 10.68 & 21.13 & 9.53 & 14.3 & 25.34 & 7.57 \\
Row 4 & 26.81 & 0 & 0.31 & 7.75 & 5.29 & 0.94 & 1.19 & 24.54 & 27.02 & 0.17 \\
Row 5 & 25.36 & 27.02 & 23.78 & 4.79 & 22.27 & 18.78 & 27.37 & 24.5 & 20.45 & 3.86 \\
Row 6 & 7.80 & 0 & 19.08 & 1.36 & 21.54 & 19.03 & 22.04 & 7.21 & 17.25 & 21.10 \\
Row 7 & 0 & 0.75 & 1.13 & 2.22 & 19.46 & 18.34 & 1.19 & 23.54 & 13.75 & 11.40 \\
Row 8 & 23.31 & 27.05 & 0.99 & 18.77 & 20.46 & 20.36 & 9.55 & 19.95 & 24.30 & 24.26 \\
Row 9 & 0 & 0 & 0 & 0 & 0 & 0 & 0 & 0 & 0 & 0 \\
Row 10 & 0 & 0 & 0 & 0 & 0 & 0 & 0 & 0 & 0 & 0 \\
\hline \multicolumn{10}{l}{} \\
\hline
\end{tabular}

Figure 11 shows the optimization process when the starting point corresponds to a configuration with all the posts introduced $28 \mathrm{~mm}$ in the filter. As it can be perceived, the optimization starts from quite a high attenuation value of $52.38 \mathrm{~dB}$ since all the posts are introduced in the filter. In this case, the GA finds much more difficult to obtain better solutions due to this highly resonant response, fact that is accentuated when all the posts are totally screwed. In any case, good attenuation values can still be found. In particular when optimizing the sixth row a value of $67.85 \mathrm{~dB}$ is obtained.

Sometimes the attenuation is reduced when optimizing the next row, since there is certain inaccuracy when changing the motorized posts from one row to the following one and when setting the optimum penetrations for the current row manually. The bandstop response of the filter is highly resonant and very sensitive to the positions of the posts, so slight deviations from the optimum positions when operating with the posts manually may lead to lower attenuation levels. This fact is intended to be minimized in the following version of the prototype by automating the process of changing the stepper motors from one row to the next one. This automation is intended to be achieved by means of a motorized system that is able to move the set of ten stepper motors along the filter in a way that no human interaction is needed.

In table 2, the optimum penetration of the posts after the optimization process when using GA starting with screwed posts is shown. Again, the screws of rows 7 to $10 \mathrm{kept}$ their initial penetration values since their optimization was not necessary.

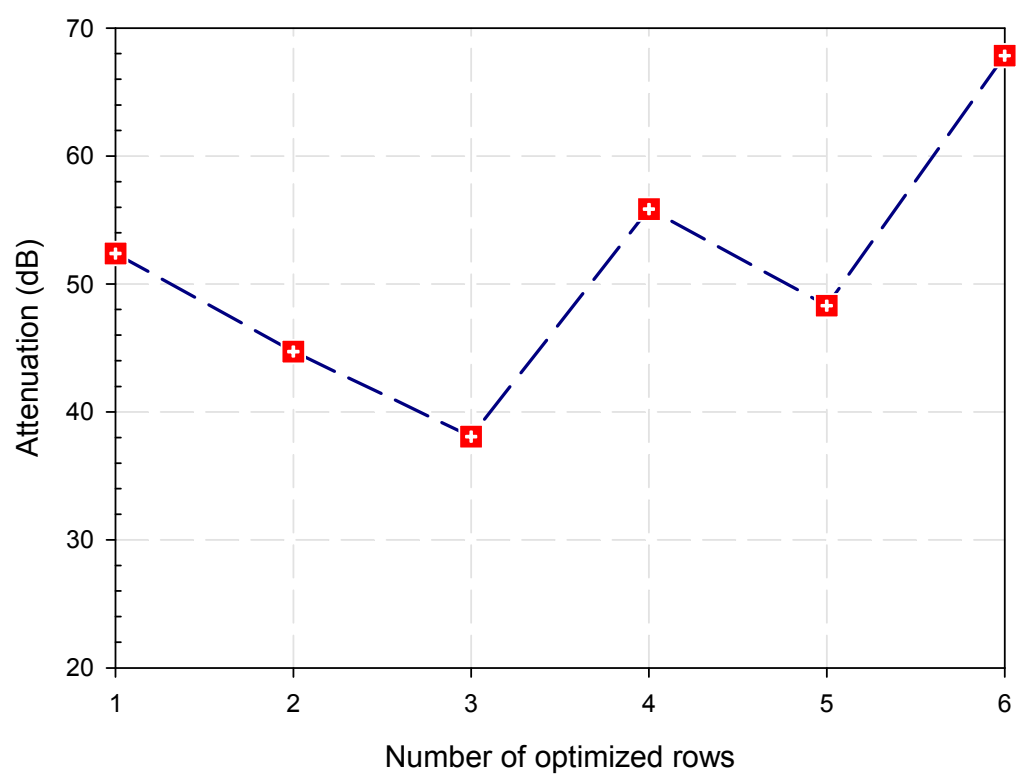

Figure 11. Attenuation in $\mathrm{dB}$ at $2.45 \mathrm{GHz}$ when using GA starting with screwed posts. 
Table 2. Penetration of the posts in $\mathrm{mm}$ when using GA starting with screwed posts.

\begin{tabular}{c|cccccccccc}
\hline \multicolumn{10}{c}{ Input port } \\
\hline & Col. 1 & Col. 2 & Col. 3 & Col. 4 & Col. 5 & Col. 6 & Col. 7 & Col. 8 & Col. 9 & Col. 10 \\
\hline Row 1 & 7.80 & 4.22 & 21.00 & 1.29 & 21.93 & 21.03 & 22.04 & 5.21 & 18.15 & 21.10 \\
Row 2 & 0 & 0.25 & 0.13 & 0.29 & 0 & 1.19 & 1.19 & 0 & 0.33 & 25.68 \\
Row 3 & 25.36 & 4.41 & 27.87 & 1.89 & 11.93 & 21.13 & 14.17 & 3.88 & 7.32 & 16.39 \\
Row 4 & 25.33 & 25.89 & 23.50 & 18.97 & 6.63 & 19.97 & 5.82 & 16.16 & 17.45 & 17.94 \\
Row 5 & 8.80 & 4.22 & 19.00 & 0.79 & 21.68 & 19.03 & 21.04 & 7.71 & 17.25 & 21.10 \\
Row 6 & 7.80 & 26.02 & 22.18 & 10.98 & 19.46 & 1.19 & 3.33 & 19.57 & 22.80 & 23.26 \\
Row 7 & 28 & 28 & 28 & 28 & 28 & 28 & 28 & 28 & 28 & 28 \\
Row 8 & 28 & 28 & 28 & 28 & 28 & 28 & 28 & 28 & 28 & 28 \\
Row 9 & 28 & 28 & 28 & 28 & 28 & 28 & 28 & 28 & 28 & 28 \\
Row 10 & 28 & 28 & 28 & 28 & 28 & 28 & 28 & 28 & 28 & 28 \\
\hline \multicolumn{10}{l}{ Output port } \\
\hline
\end{tabular}

In this way, it seems that the best option is to optimize the filter using the configuration where all posts are screwed since it starts with higher attenuation values and fewer rows are needed to be optimized to achieve the goal. Furthermore, if the oven is continuously working at high power values it would be safer to start with high attenuation values in order to reduce the energy leakage as much as possible from the beginning.

\subsection{Parametric sweeps}

In figure 12 an example of a parametric sweep of one row is shown. In particular the one corresponding to the third row during the second sweep is shown, although the other rows show similar figures only changing the position of the minimum. As it can be seen, choosing the best position for the posts is very easy just by taking the depth that obtains minimum received power at the output monopole antenna.

Figure 13 represents the attenuation for each of the three sweeps that have been carried out. It is shown a growing trend in the attenuation when incrementing the number of optimized rows as shown with the GA optimization, but also when performing more sweeps. The second and third sweeps refine the previous solutions obtaining better attenuation results. In fact, after the third sweep a good attenuation value of $62.59 \mathrm{~dB}$ is obtained.

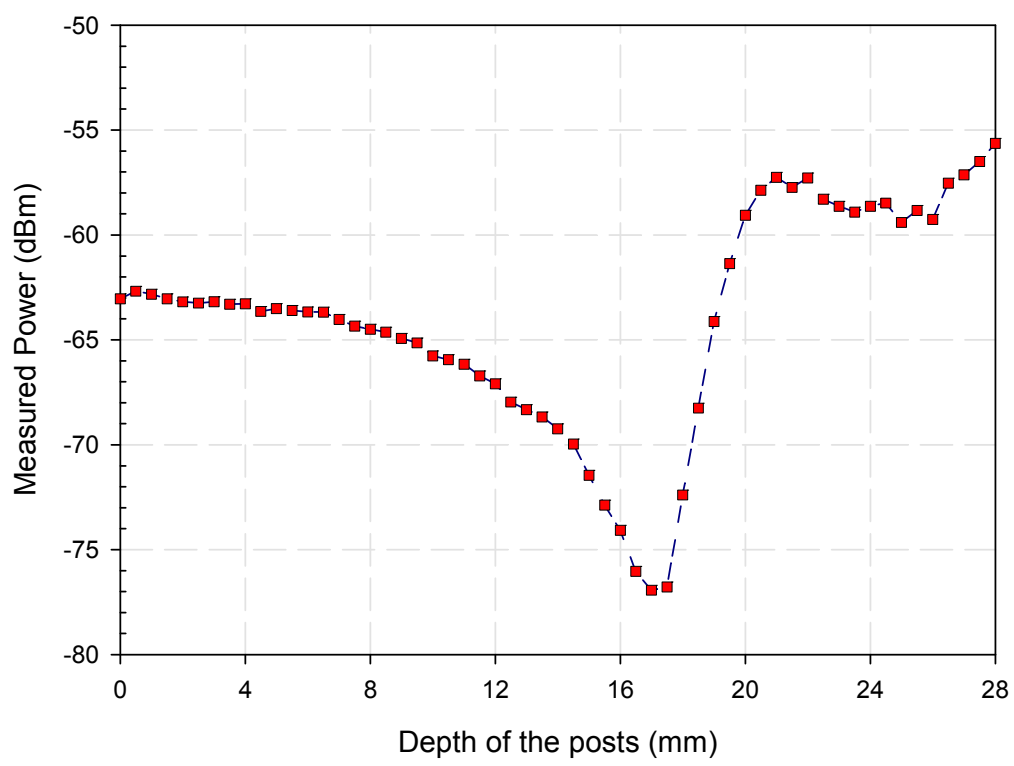

Figure 12. Example of a parametric sweep of one row of posts. 


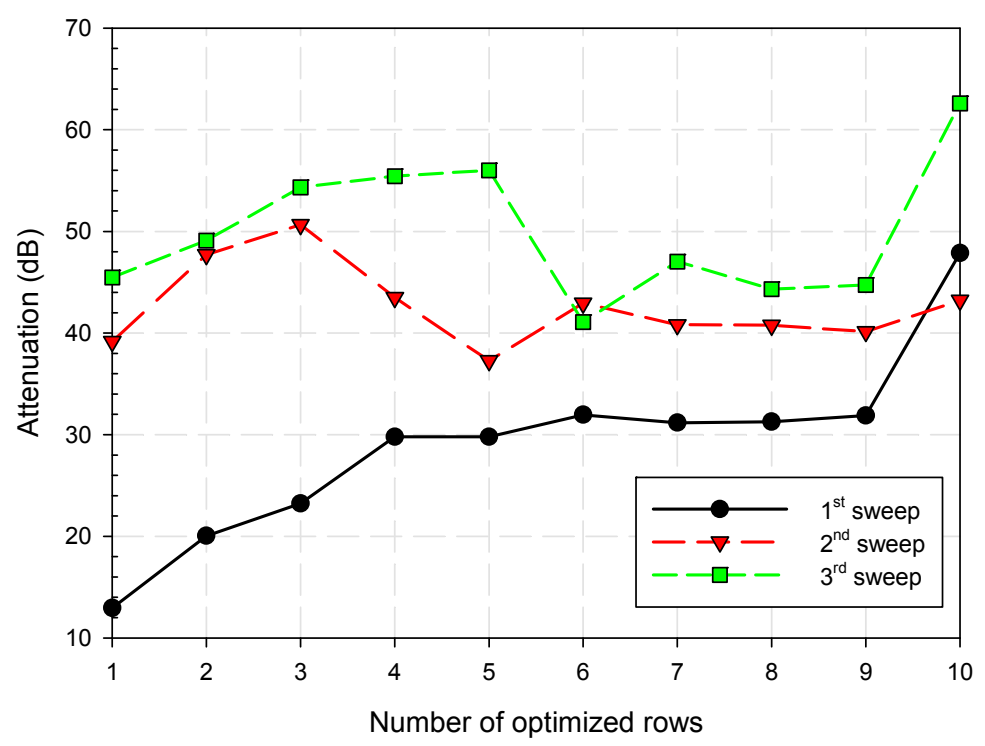

Figure 13. Attenuation in $\mathrm{dB}$ for the three parametric sweeps.

The penetration of each row of posts after each sweep is shown in table 3 .

Table 3. Penetration of each row of posts in $\mathrm{mm}$ for the three parametric sweeps.

\begin{tabular}{|c|c|c|c|}
\hline \multicolumn{4}{|c|}{ Input port } \\
\hline & ${ }^{\text {st }}$ sweep & $2^{\text {nd }}$ sweep & $3^{\text {rd }}$ sweep \\
\hline Row 1 & 26.5 & 27 & 0.5 \\
\hline Row 2 & 26.5 & 26.5 & 28 \\
\hline Row 3 & 16.5 & 17 & 15 \\
\hline Row 4 & 27 & 28 & 28 \\
\hline Row 5 & 0 & 2 & 5.5 \\
\hline Row 6 & 28 & 27 & 28 \\
\hline Row 7 & 0 & 27.5 & 28 \\
\hline Row 8 & 0 & 0.5 & 23 \\
\hline Row 9 & 8.5 & 10 & 9 \\
\hline Row 10 & 15 & 21.5 & 10 \\
\hline \multicolumn{4}{|c|}{ Output port } \\
\hline
\end{tabular}

Once the optimum configuration has been obtained after the third sweep, a measurement of the attenuation from 2.4 to $2.5 \mathrm{GHz}$ has been performed in order to see the bandwidth of the filter. As it can be seen in figure 14, the filter shows a highly resonant response since the optimization process has been performed at the single frequency of $2.45 \mathrm{GHz}$. Performing the optimization on a wider frequency range may improve the bandwidth of the filter. Nevertheless, the spectrum of a typical magnetron is quite narrow provided it is well matched.

\section{Conclusion}

In this work, a new self-configurable waveguide bandstop filter based on a combination of metallic irises and reconfigurable posts has been presented and experimentally validated. Results show that good attenuation values over $60 \mathrm{~dB}$ can be obtained for the $\mathrm{TE}_{(2 k-1) 0}$ modes. In fact, one of the main differences between the proposed filter and other automatic devices such as an auto-tuner is that the filter must deal with several propagating modes whereas the auto-tuner usually deals with the fundamental mode of the waveguide. Furthermore, although there might be some similarities between the tuning processes employed by the two devices, the goal is completely different since the auto-tuner intends to provide a good matching whereas the goal of the filter is the opposite. 


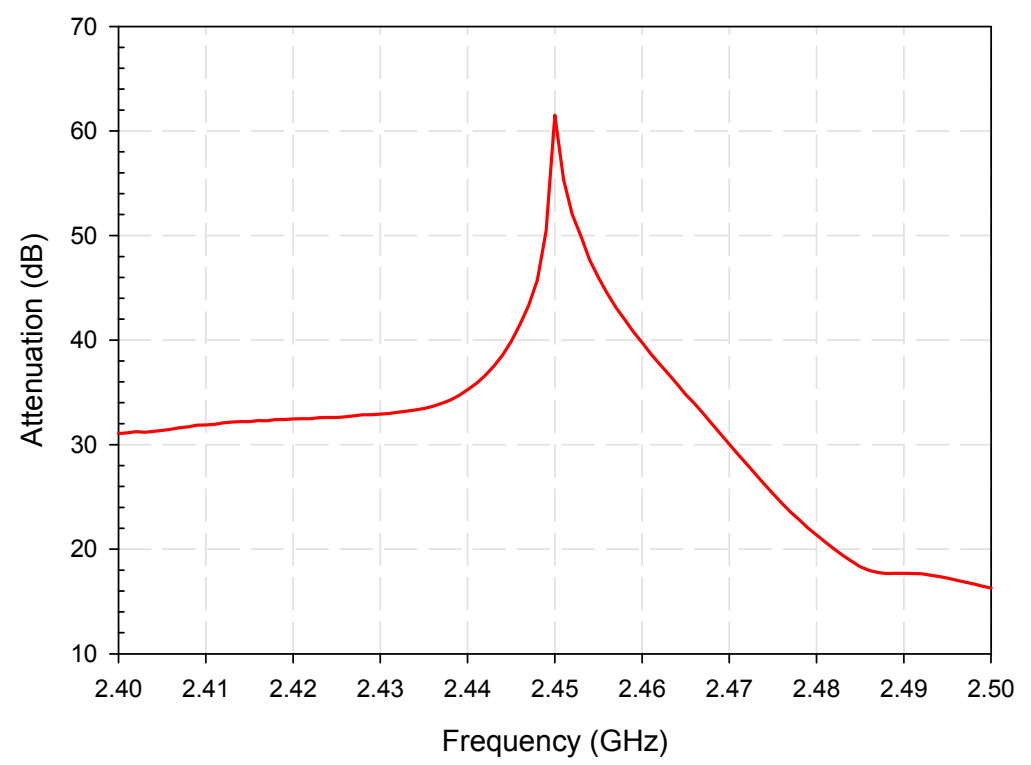

Figure 14. Attenuation in $\mathrm{dB}$ for the optimum configuration from 2.4 to $2.5 \mathrm{GHz}$.

It must be pointed out that in this work the $\mathrm{TE}_{(2 k) 0}$ modes have not been considered neither in the feeding port nor in the output port, however the flexibility of the manufactured prototype allows us to extend the work to the multimode approach. Moreover, optimizations over a wider frequency range might improve the bandwidth of the filter, so further work is envisaged in this direction.

Two different optimization and reconfiguration alternatives such as genetic algorithms and parametric sweeps have been evaluated. Regarding GA, two different alternatives have been assessed, i.e. starting the optimization process from the row nearest to the input port with all posts totally unscrewed or starting with all posts almost totally screwed and then applying a complete GA optimization stage. Results show that the best option is to optimize the filter using the configuration where all posts are screwed since it starts with higher attenuation values and fewer rows are needed to be optimized to achieve the goal. In addition, it is safer to start with high attenuation values in order to reduce the energy leakage as much as possible from the beginning.

Moreover, it has been shown that the asymmetric configurations for the metallic posts obtained by the GA can provide acceptable attenuations, which had not been proved before for this kind of filters.

Regarding the parametric sweep, it has been shown that good attenuation values can be obtained by using this approach. However, it is clear that GA are a better search strategy since the algorithm has more freedom when searching for the solution. In fact, the number of measurements that need to be done in order to achieve the goal is smaller than the case when using parametric sweeps. For instance, the number of total measurements for the GA optimization is 880 for the first scenario and 660 for the second scenario, whereas for the parametric sweep the number of total evaluations is 1710 .

Design and optimization times are also remarkably reduced when using the proposed prototype compared to the traditional approach of using simulation software. For instance, the optimization time per row when using the manufactured filter is less than an hour, whereas optimizing one row with a simulator may take several tens of hours due to the large size and complexity of the structure.

Finally, although the presented procedure is oriented to laboratory tests, it must be said that the same prototype and procedures with some minor adjustments can be applied to actual industrial microwave-heating ovens.

\section{Acknowledgement}

This work was supported in part by the Fundación Séneca, Agencia de Ciencia y Tecnología de la Región de Murcia, under the Séneca predoctoral fellowship with reference 16381/FPI/10, and under the project with reference 11689/PI/09.

\section{References}

[1] Thuery J 1992 Microwaves: Industrial, Scientific and Medical Applications (Norwood, MA: Artech House) 
[2] IEEE Std C95.1-2005 IEEE Standard for Safety Levels With Respect to Human Exposure to Radio Frequency Electromagnetic Fields, $3 \mathrm{kHz}$ to $300 \mathrm{GHz}$

[3] Levy R 2009 Compact waveguide bandstop filters for wide stopbands Microwave Symp. Digest MTT'09 IEEE MTT-S International 1245-8

[4] Vale C A W, Meyer P and Palmer K D 2000 A design procedure for bandstop filters in waveguides supporting multiple propagating modes IEEE Trans. Microwave Theory Tech. 6 2496-503

[5] Meyer P, Vale C A and Steyn W 2003 On the design of waveguide devices using multiple propagating modes 6th Int. Conf. on Telecommunications in Modern Satellite, Cable and Broadcasting Service 329-36

[6] Saxon D S and Schwinger J 1968 Discontinuities in Waveguides: Notes on Lectures by Julian Schwinger (New York, NY: Gordon and Breach Science Pub)

[7] Li P G, Adams A T, Leviatan Y and Perini J 1984 Multiple-post inductive obstacles in rectangular waveguide IEEE Trans. Microwave Theory Tech. 32 365-73

[8] Gesche R and Löchel N 1989 Two cylindrical objects in a rectangular waveguide - Resonances and filter applications IEEE Trans. Microwave Theory Tech. 37 962-8

[9] Rozzi T, Moglie F, Morini A, Gulloch W and Politi M 1992 Accurate full-band equivalent circuits of inductive posts in rectangular waveguide IEEE Trans. Microwave Theory Tech. 40 1000-9.

[10] Yao H, Zaki K A, Atia A E and Hershtig R 1995 Full wave modeling of conducting posts in rectangular waveguides and its applications to slot coupled combline filters IEEE Trans. Microwave Theory Tech. 43 2824-30

[11] Sanadi A B, Ramesh M and Kalghatgi A T 1999 Computer aided design and analysis of waveguide post filter. Asia Pacific Microwave Conf. 523-6

[12] Bekheit M, Amari S, Menzel W and Rosenberg U 2007 Design of bandstop filters using cylindrical metallic posts 37th European Microwave Conf. 870-3

[13] Li H, Wang Q, Liu R and Zhang X 2008 Economical compact waveguide band-pass filter with circular posts Int. Conf. on Microwave and Millimeter Wave Technology 318-9.

[14] Paterson N G and Anderson I 1976 Bandstop iris for rectangular waveguide Electronics Letters $12592-4$

[15] Arndt F, Duschak T, Papziner U and Rolappe P 1990 Asymmetric iris coupled cavity filters with stopband poles Microwave Symp. Digest 1990 IEEE MTT-S International 215-8.

[16] Papziner U and Arndt F 1993 Field theoretical computer-aided design of rectangular and circular iris coupled rectangular or circular waveguide cavity filters IEEE Trans. Microwave Theory Tech. 41 462-71

[17] Arndt F, Papziner U and Sieverding T 1991 Generalized S-matrix CAD of aperture coupled bandpass, bandstop, and elliptic function waveguide filters 21st European Microwave Conf. 309-14

[18] Kirilenko A A and Mos'pan L P 1998 Bandpass and bandstop multiaperture irises for millimeter and centimeter wave range MSMW'98 Symp. Proc. 352-3

[19] Yatsuk L P, Nosenko O N and Mos'pan L P 2004 Analysis and synthesis of slotted strips notch and bandstop filters with the aperture method MSMW'04 Symp. Proc. 719-21

[20] Vankouhgnett A L and Dunn J G 1973 Doubly corrugated chokes for microwave heating systems J. Microwave Power 8 101-10

[21] Soto P, Boria V E, Catalá-Civera J M, Chouaib N, Guglielmi M and Gimeno B 2000 Analysis, design, and experimental verification of microwave filters for safety issues in open-ended waveguide systems IEEE Trans. Microwave Theory Tech. 48 2133-40

[22] Catalá-Civera J M, Soto P, Boria V E, Balbastre J V and de los Reyes E 2006 Design parameters of multiple reactive chokes for open ports in microwave heating systems Advances in Microwave and Radio Frequency Processing (Berlin, Germany: Springer) pp 39-47

[23] Clemente-Fernández F J, Monzó-Cabrera J, Pedreño-Molina J L, Lozano-Guerrero A J and DíazMorcillo A 2011 Analysis of reactive and resistive open waveguide filters for use in microwave-heating applicators COMPEL: The Int. J. for Computation and Mathematics in Electrical and Electronic Engineering 30 1606-15

[24] Pedreño-Molina J L, Monzó-Cabrera J, Díaz-Morcillo A and Clemente-Fernández F J 2010 Selfconfigurable filter for continuous-flow microwave ovens, self-configurable filtering process for continuous-flow microwave ovens and continuous-flow microwave oven, ES Patent 2334549 
[25] Monzó-Cabrera J, Pedreño-Molina J L, Díaz-Morcillo A, Clemente-Fernández F J, Díaz-García J, Baños-Tudela J M and Méndez-Hernández P A 2011 Multimode open waveguide filter combining irises and posts for microwave-heating ovens, ES Patent 2337756

[26] Bilik V and Bezek J 2009 Automatic impedance matching under high power complex signal conditions Radioelektronika, 2009. 19th International Conference 141-4

[27] Computer Simulation Technology CST STUDIO SUITE ${ }^{\mathrm{TM}} \mathrm{http}: / / w w w . c s t . c o m$

[28] Bilik V and Bezek J 2010 High power limits of waveguide stub tuners J. Microwave Power 44 $178-86$

[29] MathWorks ${ }^{\circledR}$ MATLAB ${ }^{\circledR}$ http://www.mathworks.com

[30] Rahmat-Samii Y and Michielsen E 1999 Electromagnetic Optimization by Genetic Algorithms (New York: John Wiley \& Sons)

[31] Requena-Pérez M E, Albero-Ortiz A, Monzó-Cabrera J and Díaz-Morcillo A 2006 Combined use of genetic algorithms and gradient descent optimization methods for accurate inverse permittivity measurement IEEE Trans. Microwave Theory Tech. 54 615-24

[32] Lozano-Guerrero A, Clemente-Fernández F J, Monzó-Cabrera J, Pedreño-Molina J L and DíazMorcillo A 2010 Precise evaluation of coaxial to waveguide transitions by means of inverse techniques IEEE Trans. Microwave Theory Tech. 58 229-35 